\title{
Two new 2-alkylquinolones, inhibitory to the fish skin ulcer pathogen Tenacibaculum maritimum, produced by a rhizobacterium of the genus Burkholderia sp.
}

\author{
Dandan $\mathrm{Li}^{1}$, Naoya Oku${ }^{1}$, Atsumi Hasada ${ }^{1}$, Masafumi Shimizu ${ }^{2}$ and Yasuhiro Igarashi ${ }^{* 1}$
}

\section{Letter}

\section{Address:}

${ }^{1}$ Biotechnology Research Center and Department of Biotechnology,

Toyama Prefectural University, 5180 Kurokawa, Imizu, Toyama 939-0398, Japan and 2Laboratory of Plant Pathology, Faculty of Applied Biological Sciences, Gifu University, 1-1 Yanagido, Gifu 501-1193, Japan

Email:

Yasuhiro Igarashi* - yas@pu-toyama.ac.jp

* Corresponding author

Keywords:

antimicrobial; Burkholderia; quinolone; skin ulcer; Tenacibaculum maritimum

\author{
Beilstein J. Org. Chem. 2018, 14, 1446-1451. \\ doi:10.3762/bjoc. 14.122 \\ Received: 29 March 2018 \\ Accepted: 22 May 2018 \\ Published: 14 June 2018 \\ Associate Editor: J. S. Dickschat \\ (C) $2018 \mathrm{Li}$ et al.; licensee Beilstein-Institut. \\ License and terms: see end of document.
}

\begin{abstract}
Exploration of rhizobacteria of the genus Burkholderia as an under-tapped resource of bioactive molecules resulted in the isolation of two new antimicrobial 2-alkyl-4-quinolones. (E)-2-(Hept-2-en-1-yl)quinolin-4(1H)-one (1) and (E)-2-(non-2-en-1-yl)quinolin4(1H)-one (3) were isolated from the culture broth of strain MBAF1239 together with four known alkylquinolones (2 and 4-6), pyrrolnitrin (7), and BN-227 (8). The structures of $\mathbf{1}$ and $\mathbf{3}$ were unambiguously characterized using NMR spectroscopy and mass spectrometry. Compounds 1-8 inhibited the growth of the marine bacterium Tenacibaculum maritimum, an etiological agent of skin ulcers in marine fish, offering new opportunities to develop antibacterial drugs for fish farming.
\end{abstract}

\section{Findings}

Bacteria of the genus Burkholderia within the family Burkholderiaceae [1], along with their neighboring genera, Paraburkholderia, Caballeronia [2], and Robbsia [3] constitute a distinct group of bacteria within the class Betaproteobacteria. These bacteria are obligatory aerobic, mostly motile, non-sporeforming Gram-negative rods of strictly terrestrial origin. Although isolation of Burkholderia from marine sediments has been reported, these bacteria may not originate in the marine

environment, as enrichment in low salinity media is a prerequisite for isolation [4-6]. Most of them live in close association with animals or plants as pathogens or symbionts and exhibit a variety of catabolic and metabolic activities $[1,7]$.

One hundred ten secondary metabolites have been reported from Burkholderia (data retrieved from the Dictionary of Natural Products, as of March 20, 2018). However, it is likely 
that Burkholderia produce many more secondary metabolites than reported, as this group was previously classified into the genus Pseudomonas [8]. In fact, the high capacity of Burkholderia in secondary metabolism is demonstrated by the presence of unique functionalities, such as monocyclic 3-pyrazolone [9], $\alpha$-aminoacrylonitrile, and thioimidazolinone [10], all of which are not preceded in metabolites from other taxa.

The large genome size of Burkholderia also suggests a high capacity for secondary metabolism. According to the NCBI genome database (https://www.ncbi.nlm.nih.gov/genome/ browse\#!/prokaryotes/), the genome sizes of $B$. cepacia ATCC25416, Paraburkholderia terrae DSM 17804, and Caballeronia glathei DSM50014 are 8.61, 10.1, and 8.64 Mbp, respectively, which are comparable to $9.05 \mathrm{Mbp}$ for Streptomyces coelicolor A3(2) and 9.14 Mbp for Myxococcus xanthus DK 1622, both known as representatives of prolific antibiotic producers (as of Jan. 20, 2018).

As part of our program to further explore this unique pharmacological resource, rhizobacteria of the genus Burkholderia were collected and tested for the production of antimicrobial metabolites against a panel of plant and animal pathogens consisting of 4 bacteria, 1 yeast, and 4 fungi. The result of this screening prompted the detailed chemical study of a strain coded as MBAF1239, which resulted in the isolation of eight antibacterial metabolites, including the two new 2-alkylquinolones $\mathbf{1}$ and $\mathbf{3}$ (Figure 1).

Strain MBAF1239 was seed-cultured in V22 medium and subsequently transferred into IMM-HS medium, which was designed for metabolite production based on the composition of IMM [11] and HS media [12]. The fermented culture was extracted with $1-\mathrm{BuOH}$ and the extract was fractionated by solvent-partitioning to yield $n$-hexane-, $90 \%$ aqueous $\mathrm{MeOH}$-, and $60 \%$ aqueous $\mathrm{MeOH}$-soluble fractions. Antimicrobial testings against Rhizopus oryzae (the fungal pathogen of rice seedling blight), Trichophyton rubrum (dermatophytosis pathogen), and Tenacibaculum maritimum (the causative organism for skin ulcers in marine fish) revealed that the second fraction was the most active. The second fraction was then further fractionated by ODS flash chromatography and purified by HPLC to yield the eight metabolites $\mathbf{1}-\mathbf{8}$.

The molecular formula of $\mathbf{3}$ was established as $\mathrm{C}_{18} \mathrm{H}_{23} \mathrm{NO}$ based on a HRESITOFMS measurement $(\mathrm{m} / \mathrm{z} 270.1855$ $\left.[\mathrm{M}+\mathrm{H}]^{+}, \Delta+0.3 \mathrm{mmu}\right)$. Analysis of ${ }^{1} \mathrm{H},{ }^{13} \mathrm{C}$, and HSQC NMR spectra in $\mathrm{CDCl}_{3}$ (Supporting Information File 1, Figures S6, S7, and S9) revealed four aromatic $\left(\delta_{\mathrm{H}} / \delta_{\mathrm{C}} 8.34 / 126.3,7.57 /\right.$ $131.9,7.32 / 123.6$, and $7.26 / 116.8)$ and three olefinic $\left(\delta_{\mathrm{H}} / \delta_{\mathrm{C}}\right.$ $6.18 / 109.5,5.79 / 137.7$, and 5.54/123.0) methines, six aliphatic methylenes $\left(\delta_{\mathrm{H}} / \delta_{\mathrm{C}} 3.37 / 37.4,2.12 / 32.5,1.43 / 29.1,1.33 / 28.9\right.$, $1.31 / 22.6$, and $1.30 / 31.7)$, and a methyl $\left(\delta_{\mathrm{H}} / \delta_{\mathrm{C}} 0.89 / 14.1\right)$ group, leaving one carbonyl $\left(\delta_{\mathrm{C}} 179.0\right)$ and three aromatic resonances $\left(\delta_{\mathrm{C}} 149.9,139.4\right.$, and 125.3$)$ as quaternary carbons (Table 1). Because these structural elements accounted for six out of eight degrees of unsaturation, the remaining two degrees correspond to two rings, which constitutes a fused bicyclic structure as suggested by the number of available aromatic carbons (eleven). A 4-quinolone substructure was indicated by a peaksplitting at the $340-320 \mathrm{~nm}$ region in the UV spectrum (328 and $322 \mathrm{~nm}$ ) [13]. Indeed, ${ }^{1} \mathrm{H}$ NMR resonances at the down field region was superimposable on those of known 2-heptyl-4(1H)quinolone (2, Supporting Information File 1, Figure S11). The COSY and HMBC correlations also supported this assignment (Figure 2; Supporting Information File 1, Figures S8 and S10). An extension of a 2-nonenyl group (C9-C17) at C2 was supported by an $\mathrm{HMBC}$ correlation from $\mathrm{H} 3$ to $\mathrm{C} 9$ (Figure 2).

The obtuse ${ }^{1} \mathrm{H}$ NMR signal shapes, presumably due to limited solubility of $\mathbf{3}$ in $\mathrm{CDCl}_{3}$, hampered unequivocal determination of the C10-geometry based on a coupling constant between the olefinic protons H10 and H11. Instead, a lack of NOESY correlation between these protons was indicative of an $E$-geometry (Supporting Information File 1, Figure S12). This was finally supported by a chemical shift value for the $\mathrm{C} 12$ allylic carbon at $\delta 32.5$, which is closer to that of an $E$-isomer, burkholone $(\delta 32.5)$ [14], than that of an $Z$-isomer, haplacutine $F(\delta 27.7)$
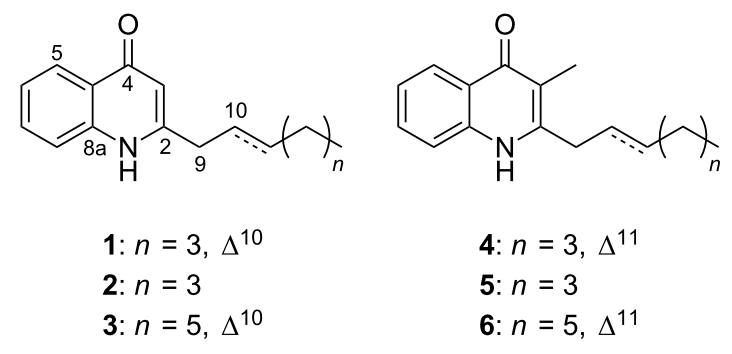<smiles>O=[N+]([O-])c1c(Cl)cccc1-c1c[nH]cc1Cl</smiles>

7<smiles>COc1ccc(=O)n(O)c1C</smiles>

8

Figure 1: Structures of compounds 1-8. 
Table 1: NMR data for $(E)-2$-(non-2-en-1-yl)quinolin-4(1H)-one (3) in $\mathrm{CDCl}_{3}$ ( $\delta$ in ppm).

\begin{tabular}{lllll} 
pos. & $\delta_{\mathrm{C}}$ & $\begin{array}{l}\delta_{\mathrm{H}}, \text { mult. }(\mathrm{J} \text { in } \\
\mathrm{Hz}), \text { integr. }\end{array}$ & $\mathrm{COSY}$ & $\begin{array}{l}\mathrm{HMBC}\left({ }^{1} \mathrm{H} \text { to }\right. \\
\left.{ }^{13} \mathrm{C}\right)\end{array}$ \\
\hline 1 & \multicolumn{5}{c}{$8.07, \mathrm{br}, 1 \mathrm{H}$} \\
2 & 149.9 & & \\
3 & 109.5 & $6.18, \mathrm{~s}, 1 \mathrm{H}$ & & $2,4 \mathrm{a}, 9$ \\
4 & 179.0 & & \\
$4 \mathrm{a}$ & 125.3 & & & \\
5 & 126.3 & $8.34, \mathrm{~d}(7.9), 1 \mathrm{H}$ & 6 & $4,7,8 \mathrm{a}$ \\
6 & 123.6 & $7.32, \mathrm{t}, 7.5,1 \mathrm{H}$ & 5,7 & $4 \mathrm{a}, 8$ \\
7 & 131.9 & $7.57, \mathrm{brs}, 1 \mathrm{H}$ & 6 & \\
8 & 116.8 & $7.26, \mathrm{ovl}$ & & \\
$8 \mathrm{a}$ & 139.4 & & & \\
9 & 37.4 & $3.37, \mathrm{brs}, 2 \mathrm{H}$ & 10 & \\
10 & 123.0 & $5.54, \mathrm{~m}, 1 \mathrm{H}$ & 9,11 & \\
11 & 137.7 & $5.79, \mathrm{~m}, 1 \mathrm{H}$ & 10,12 & \\
12 & 32.5 & $2.12 \mathrm{ddd}(7.2$, & 11,13 & $10,11,13,14$ \\
& & $6.5,6.3), 2 \mathrm{H}$ & & \\
13 & 29.1 & $1.43, \mathrm{~m}, 2 \mathrm{H}$ & 12,14 & \\
14 & 28.9 & $1.33, \mathrm{~m}, 2 \mathrm{H}$ & & \\
15 & 31.7 & $1.30, \mathrm{~m}, 2 \mathrm{H}$ & & \\
16 & 22.6 & $1.31, \mathrm{~m}, 2 \mathrm{H}$ & 17 & \\
17 & 14.1 & $0.89, \mathrm{t}(6.5), 3 \mathrm{H}$ & 16 & 15,16 \\
\hline
\end{tabular}

asignal overlapped by a residual solvent peak.

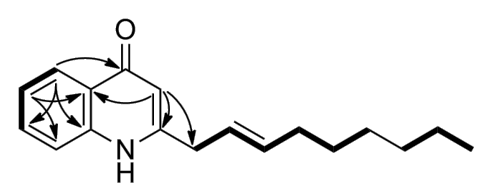

Figure 2: Key COSY (bold line) and HMBC (arrow) correlations for 3

[15] (Figure 3). Thus, the structure of $\mathbf{3}$ was concluded to be (E)-2-(non-2-en-1-yl)quinolin-4(1H)-one.

The molecular ions of 1 were observed at $\mathrm{m} / \mathrm{z} 242$ and $\mathrm{m} / \mathrm{z} 240$ in the positive and negative modes, respectively, revealing a $28 \mathrm{Da}$ smaller molecular weight for 1 relative to 3 . The ${ }^{1} \mathrm{H}$ NMR spectra of both compounds (Supporting Information File 1, Figures S6 and S1) are similar except for the integral of the methylene resonances between 1.48 and $1.23 \mathrm{ppm}$. While the resonance amounted to $8 \mathrm{H}$-equivalent in $\mathbf{3}$, the resonance in $\mathbf{1}$ was equivalent to $4 \mathrm{H}$, suggesting that $\mathbf{1}$ is a congener of $\mathbf{3}$ with a two-methylene shorter appendage. This was later confirmed by the interpretation of COSY, HSQC, and HMBC spectra (Supporting Information File 1, Figures S3-S5), allowing the full assignment of ${ }^{1} \mathrm{H}$ and ${ }^{13} \mathrm{C}$ NMR resonances (see Experimental). Thus, 1 was determined to be (E)-2-(hept-2-en-1yl)quinolin-4(1H)-one.

The remaining metabolites were identified as known compounds based on NMR and MS data (data not shown). Compound 2 was identified as Pyo Ib or 2-heptylquinolin-4(1H)-one [16], 4 was identified as (E)-2-(hept-2-en-1-yl)-3-methylquinolin-4(1H)-one [13], 5 was identified as PSC-C or 2-heptyl3-methylquinolin-4(1H)-one [17], 6 was identified as PSC-D or (E)-3-methyl-2-(non-2-en-1-yl)quinolin-4(1H)-one) [17], 7 was identified as pyrrolnitrin [18], and $\mathbf{8}$ was identified as BN-227 [19] (Figure 1).

4-Quinolone is a common core in synthetic antibactericides [20] and in bioactive metabolites produced by Rutaceae plants [2125], Gram-positive [26,27] and Gram-negative bacteria [28-30], and a marine sponge [31]. Among a variety of substituted 4-quinolones, 2-alkyl-4-quinolones are the most common core in antibiotics [32], which were originally discovered as antianthrax metabolites produced by Pseudomonas aeruginosa. A series of chemoecological studies of $P$. aeruginosa has uncovered multifunctional roles of this quinolone class as antibacterial, antifungal, iron-chelating, and autoinducer agents to assist the survival of the producing organisms [32]. Additionally, drug discovery attempts have revealed 5-lipoxygenase inhibitory activity [33], plant-growth promoting activity [32,34], and IGFdependent cell-specific cytotoxicity [14].

A recent metabolomic analysis using LC-MS verified the presence of more than 50 2-alkyl-4-quinolones [35], most of which remain chemically and biologically uncharacterized. Compounds $\mathbf{1}$ and $\mathbf{3}$ are among these uncharacterized analogs, with their (presumable) detection by mass spectrometry reported twice [29,36] and three times [36-38], respectively. In this study, we have isolated both compounds for the first time,

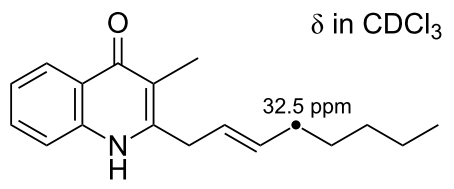

burkholone

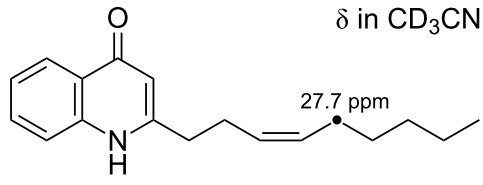

haplacutine F

Figure 3: Referential ${ }^{13} \mathrm{C}$ chemical shifts of an allylic carbon in burkholone [14] and haplacutine $\mathrm{F}$ [15]. 
which enabled rigorous structure characterization, including the position and geometry of unsaturation in the side chains, as well as evaluation of their bioactivity (see below).

Compounds 1-8 at $10 \mu \mathrm{g} / \mathrm{disc}$ inhibited the growth of a bacterium of the phylum Bacteroidetes, T. maritimum (Table 2). Overall, alkylquinolones 1-6 were more potent than 7 and 8 . Among 1-6, the 2-heptenyl-3-methyl congener 4 was the most active. Compounds 3-6 also inhibited the growth of the fungi $R$. oryzae and T. rubrum, while $\mathbf{1}$ and $\mathbf{2}$ did not. This may to some extent attributable to the global lipophilicity of molecules, as $\mathbf{1}$ and $\mathbf{2}$ are among the fastest eluting congeners during the reversed-phase separation. Because T. maritimum is one of the major etiologies for fatal skin ulcers in marine fish [39], 1-8 could offer novel scaffolds to develop new therapeutic modalities for this economically devastating epizootic.

\begin{tabular}{|c|c|c|c|}
\hline & $\begin{array}{l}\text { Tenacibaculum } \\
\text { maritimum }\end{array}$ & $\begin{array}{l}\text { Trichophyton } \\
\text { rubrum }\end{array}$ & $\begin{array}{l}\text { Rhizopus } \\
\text { oryzae }\end{array}$ \\
\hline 1 & $33^{a}$ & 0 & 0 \\
\hline 2 & 24 & 0 & 0 \\
\hline 3 & 25 & 30 & 10 \\
\hline 4 & 55 & 34 & 10 \\
\hline 5 & 22 & 20 & 30 \\
\hline 6 & 25 & 30 & 15 \\
\hline 7 & 20 & $-^{b}$ & - \\
\hline 8 & 7 & - & - \\
\hline
\end{tabular}

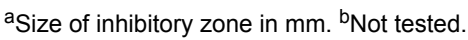

\section{Experimental}

\section{General experimental procedures}

UV and IR spectra were recorded on a Hitachi U-3210 and a Perkin Elmer Spectrum 100 spectrophotometer, respectively. ${ }^{1} \mathrm{H}$ and ${ }^{13} \mathrm{C}$ NMR spectra were obtained on a Bruker AVANCE 500 spectrometer referencing solvent peaks at $\delta_{\mathrm{H}} / \delta_{\mathrm{C}}$ 7.26/77.0 ppm for $\mathrm{CDCl}_{3}$ and $\delta_{\mathrm{H}} / \delta_{\mathrm{C}} 3.30 / 49.0 \mathrm{ppm}$ for $\mathrm{CD}_{3} \mathrm{OD}$. ESITOFMS spectra were collected on a Bruker micrOTOF focus mass spectrometer.

\section{Collection of Burkholderia strains and broth screening}

Burkholderia strains were collected by serial dilution plating on Pseudomonas agar supplemented with C-F-C (Oxoid, Basingstoke, England) from rhizosphere soils of Welsh onion and cucumber (Cucumis sativus), grown in an experimental farm at Mie University (Kurimamachiya-cho, Tsu, Mie) in 2010. The bacterial collection was cultured in 4 different media to give 152 extracts, which were screened against Edwardsiella ictaluri NBRC105724 ${ }^{\mathrm{T}}$ (pathogen of enteric septicemia of freshwater fish), T. maritimum NBRC16015, Trichophyton rubrum NBRC5467, Candida albicans NBRC0197 (human opportunistic pathogen), R. oryzae NBRC4705, Glomerella cingulata NBRC5907 (pathogen of anthracnose), Ralstonia solanacearum SUPP1541 (pathogen of bacterial wilt of Solanaceous plants), Rhizobium radiobacter NBRC14554 (pathogen of crown gall), Athelia rolfsii NBRC30071 (pathogen of southern blight). All but two strains, or 139 extracts out of 152 , exhibited activity against at least one pathogen, demonstrating an impressively high incidence of antagonistic strains in this genus. One of the prominent hit extracts was prepared from a culture of strain MBAF1239, isolated from a rhizosphere of Welsh onion (Allium fistulosum). A 16S rDNA sequence analysis identified MBAF1239 as Burkholderia sp. within the B. cepacia complex (DDBJ accession number LC194190).

\section{Fermentation, extraction, and isolation}

Burkholderia sp. MBAF1239 was seed-cultured in $500 \mathrm{~mL} \mathrm{K-1}$ flasks each containing $100 \mathrm{~mL}$ of medium V-22 (soluble starch $1 \%$, glucose $0.5 \%$, NZ-case $0.3 \%$, yeast extract $0.2 \%$, tryptone $0.5 \%, \mathrm{~K}_{2} \mathrm{HPO}_{4} 0.1 \%, \mathrm{MgSO}_{4} \cdot 7 \mathrm{H}_{2} \mathrm{O} 0.05 \%$, and $\mathrm{CaCO}_{3} 0.3 \%$, $\mathrm{pH} 7.0$ ) by rotary shaking at $200 \mathrm{rpm}$ at $30{ }^{\circ} \mathrm{C}$ for two days. A three-mL aliquot of the resulting culture was inoculated into $100 \mathrm{~mL}$ of the IMM-HS production medium (glucose $1 \%$, $\mathrm{K}_{2} \mathrm{HPO}_{4} \quad 0.36 \%, \mathrm{KH}_{2} \mathrm{PO}_{4} \quad 0.41 \%, \mathrm{MgSO}_{4} \cdot 7 \mathrm{H}_{2} \mathrm{O} \quad 0.02 \%$, $\mathrm{CaCl}_{2} \cdot 2 \mathrm{H}_{2} \mathrm{O} 0.01 \%, \mathrm{FeSO}_{4} \cdot 7 \mathrm{H}_{2} \mathrm{O} 0.002 \%, \mathrm{NH}_{4} \mathrm{Cl} 0.1 \%$, biotin $0.0001 \%$, and L-histidine $0.4 \%$ ), and shaken at $200 \mathrm{rpm}$ at $30{ }^{\circ} \mathrm{C}$ for 4 days.

For the extraction of secondary metabolites, $100 \mathrm{~mL}$ of 1-butanol was added to each flask, and they were allowed to shake for $1 \mathrm{~h}$. The resulting suspension was centrifuged at $6000 \mathrm{rpm}$ for $10 \mathrm{~min}$ to separate organic and aqueous layers, the former of which was concentrated in vacuo to give a $5.35 \mathrm{~g}$ extract from a $2 \mathrm{~L}$ culture. The crude extract was successively partitioned between $60 \% \mathrm{MeOH}(250 \mathrm{~mL})$ and $\mathrm{CH}_{2} \mathrm{Cl}_{2}(250 \mathrm{~mL} \times 3)$ and the latter between $90 \%$ aqueous $\mathrm{MeOH}(150 \mathrm{~mL})$ and $n$-hexane $(150 \mathrm{~mL} \times 3)$. The aqueous $\mathrm{MeOH}$ layer, which concentrated the antibacterial activity against Tenacibaculum maritimum, was subjected to ODS flash chromatography $(\varnothing 3 \times 7 \mathrm{~cm})$ eluted with a stepwise gradient of $25,40,55$, and $85 \%(\mathrm{v} / \mathrm{v})$ $\mathrm{MeCN}$ in $50 \mathrm{mM} \mathrm{NaClO}$. The most active third fraction (37.4 mg) was purified by reversed-phase HPLC on a Cosmosil AR-II column $(\varnothing 1 \times 25 \mathrm{~cm})$ with a linear gradient elution program [eluents: $\mathrm{MeOH}(\mathrm{A}), 1: 1 \mathrm{CH}_{3} \mathrm{CN} / \mathrm{H}_{2} \mathrm{O}$ (B); 0-5 min $100 \% \mathrm{~B}, 5-45 \min 100 \% \mathrm{~B}$ to $0 \% \mathrm{~B}, 45-65 \min 0 \% \mathrm{~B}$; flow $3 \mathrm{~mL} \mathrm{~min}-1$; UV detection at $210 \mathrm{~nm}$ ] to afford two new 2-alkylquinolones $\mathbf{1}(0.5 \mathrm{mg})$ and $\mathbf{3}(0.7 \mathrm{mg})$, together with four known 2-alkylquinolones, Pyo Ib or 2-heptylquinolin-4(1H)one (2, $2.3 \mathrm{mg}),(E)$-2-(hept-2-en-1-yl)-3-methylquinolin- 
4(1H)-one $(4,2.5 \mathrm{mg})$, PSC-C or 2-heptyl-3-methylquinolin4(1H)-one $(5,1.0 \mathrm{mg})$, PSC-D or $(E)$-3-methyl-2-(non-2-en-1yl)quinolin-4(1H)-one $(6,0.8 \mathrm{mg})$, pyrrolnitrin $(7,0.5 \mathrm{mg})$, and $\mathrm{BN}-227$ (8, $0.8 \mathrm{mg})$.

(E)-2-(Hept-2-en-1-yl)quinolin-4(1H)-one (1): UV (MeCN) $\lambda_{\max }, \mathrm{nm}(\varepsilon): 328$ (29900), 322 (27600), 316 (29800), 292 (10700), 288 (10900), 260 (6600), 240 (51200); IR $v_{\max }$ (ATR) $\mathrm{cm}^{-1}: 2927,2873,1636,1595,1553,1505,1473,1445,1355$, 1322, 1275, 1104, 1028, 969, 841, 762, 676; ${ }^{1} \mathrm{H}$ NMR $\left(\mathrm{CD}_{3} \mathrm{OD}\right) \delta_{\mathrm{H}} 8.20(\mathrm{~d}, J=8.1 \mathrm{~Hz}, 1 \mathrm{H}, \mathrm{H} 5), 7.68$ (brs, $\left.1 \mathrm{H}, \mathrm{H} 7\right)$, 7.57 (brs, 1H, H8), 7.39 (brt, $J=6.9 \mathrm{~Hz} 1 \mathrm{H}, \mathrm{H} 6$ ), 6.21 (s, 1H, H3), 5.71 (m, 1H, H11), 5.61 (brd, $J=13.9 \mathrm{~Hz}, 1 \mathrm{H}, \mathrm{H} 10), 3.42$ (brs, 2H, H9), 2.09 (dt, $J=6.3$ and $6.2 \mathrm{~Hz}, 2 \mathrm{H}, \mathrm{H} 12), 1.38$ (m, 2H, H13), 1.34 (m, 2H, H14), 0.90 (t, $J=6.9 \mathrm{~Hz}, 3 \mathrm{H}, \mathrm{H} 15) ;{ }^{13} \mathrm{C}$ NMR $\left(\mathrm{CD}_{3} \mathrm{OD}\right) \delta_{\mathrm{C}} 180.8(\mathrm{C} 4), 155.7(\mathrm{C} 2), 141.6(\mathrm{C} 8 \mathrm{a}), 136.5$ (C11), 133.5 (C7), 126.0 (C5), 125.5 (C4a), 125.3 (C10), 125.1 (C6), 119.1 (C8), 108.9 (C3), 37.8 (C9), 33.2 (C12), 32.6 (C13), 23.2 (C14), 14.2 (C15); ESIMS-TOF $(\mathrm{m} / \mathrm{z}):[\mathrm{M}+\mathrm{H}]^{+}$calcd for $\mathrm{C}_{16} \mathrm{H}_{19} \mathrm{NO}$, 242.1539; found, 242.1539.

(E)-2-(Non-2-en-1-yl)quinolin-4(1H)-one (3): UV (MeCN) $\lambda_{\max }, \mathrm{nm}(\varepsilon): 328$ (18600), 322 (16800), 316 (18200), 292 (8000), 288 (8400), 260 (5300), 240 (23,200); IR $v_{\max }$ (ATR) $\mathrm{cm}^{-1}: 2923,2853,1730,1635,1593,1554,1500,1471,1443$, $1354,1320,1247,1137,1028,965,836,759,672$; HRESIMS-TOF $(\mathrm{m} / z):[\mathrm{M}+\mathrm{H}]^{+}$calcd for $\mathrm{C}_{18} \mathrm{H}_{24} \mathrm{NO}$, 270.18524 ; found, 270.1855 .

\section{Evaluation of antimicrobial activity}

The antibacterial and antifungal activity of $\mathbf{1}-\mathbf{8}$ was evaluated by a paper-disc agar diffusion method described in our previous study [40]. Flexibacter maritimus medium ( $0.5 \%$ peptone and $0.05 \%$ yeast extract in sea water) solidified with $10 \%$ agar was used to test against T. maritimum.

\section{Supporting Information}

$$
\begin{aligned}
& \text { Supporting Information File } 1 \\
& { }^{1} \mathrm{H} \text { and }{ }^{13} \mathrm{C} \text { NMR, COSY, HSQC, and HMBC spectra for } \\
& \text { compounds } \mathbf{1} \text { and } \mathbf{3} \text {. } \\
& \text { [https://www.beilstein-journals.org/bjoc/content/ } \\
& \text { supplementary/1860-5397-14-122-S1.pdf] }
\end{aligned}
$$

\section{Acknowledgements}

We thank Prof. Yasufumi Hikichi and Dr. Ayami Kanda at Kochi University for providing $R$. solanacearum SUPP1541. This work was supported in part by JSPS KAKENHI Grant Number JP26660096 and President's Fund Initiative at Toyama Prefectural University.

\section{ORCID ${ }^{\circledR}$ iDs}

Naoya Oku - https://orcid.org/0000-0002-2171-2168

\section{References}

1. Depoorter, E.; Bull, M. J.; Peeters, C.; Coenye, T.; Vandamme, P.; Mahenthiralingam, E. Appl. Microbiol. Biotechnol. 2016, 100, 5215-5229. doi:10.1007/s00253-016-7520-x

2. Estrada-de los Santos, P.; Rojas-Rojas, F. U.; Tapia-García, E. Y.; Vásquez-Murrieta, M. S.; Hirsch, A. M.

Ann. Microbiol. (Heidelberg, Ger.) 2016, 66, 1303-1314. doi:10.1007/s13213-015-1183-1

3. Beukes, C. W.; Palmer, M.; Manyaka, P.; Chan, W. Y.; Avontuur, J. R.; van Zyl, E.; Huntemann, M.; Clum, A.; Pillay, M.; Palaniappan, K.; Varghese, N.; Mikhailova, N.; Stamatis, D.; Reddy, T. B. K.; Daum, C.; Shapiro, N.; Markowitz, V.; Ivanova, N.; Kyrpides, N.; Woyke, T.; Blom, J.; Whitman, W. B.; Venter, S. N.; Steenkamp, E. T. Front. Microbiol. 2017, 8, No. 1154. doi:10.3389/fmicb.2017.01154

4. Rusch, A.; Islam, S.; Savalia, P.; Amend, J. P. Int. J. Syst. Evol. Microbiol. 2015, 65, 189-194. doi:10.1099/ijs.0.064477-0

5. Revathy, T.; Jayasri, M. A.; Suthindhiran, K. Scientifica 2015, No. 867586. doi:10.1155/2015/867586

6. Wang, Y.; Yin, B.; Hong, Y.; Yan, Y.; Gu, J.-D. Ecotoxicology 2008, 17, No. 845. doi:10.1007/s10646-008-0247-4

7. Suárez-Moreno, Z. R.; Caballero-Mellado, J.; Coutinho, B. G.; Mendonça-Previato, L.; James, E. K.; Venturi, V. Microb. Ecol. 2012, 63, 249-266. doi:10.1007/s00248-011-9929-1

8. Yabuuchi, E.; Kosako, Y.; Oyaizu, H.; Yano, I.; Hotta, H.; Hashimoto, Y.; Ezaki, T.; Arakawa, M. Microbiol. Immunol. 1992, 36, 1251-1275. doi:10.1111/j.1348-0421.1992.tb02129.x

9. Mitchell, R. E.; Greenwood, D. R.; Sarojini, V. Phytochemistry 2008, 69, 2704-2707. doi:10.1016/j.phytochem.2008.08.013

10. Brady, S. F.; Bauer, J. D.; Clarke-Pearson, M. F.; Daniels, R. J. Am. Chem. Soc. 2007, 129, 12102-12103. doi:10.1021/ja075492v

11. Ballio, A.; Barra, D.; Bossa, F.; DeVay, E. J.; Grgurina, I.; lacobellis, N. S.; Marino, G.; Pucci, P.; Simmaco, M.; Surico, G. Physiol. Mol. Plant Pathol. 1988, 33, 493-496. doi:10.1016/0885-5765(88)90014-8

12. Hoitink, H. A. J.; Sinden, S. L. Phytopathology 1970, 60, 1236-1237. doi:10.1094/Phyto-60-1236

13. Hashimoto, M.; Hattori, K. Chem. Pharm. Bull. 1967, 15, 718-720. doi:10.1248/cpb.15.718

14. Mori, T.; Yamashita, T.; Furihata, K.; Nagai, K.; Suzuki, K.-i.; Hayakawa, Y.; Shin-ya, K. J. Antibiot. 2007, 60, 713-716. doi:10.1038/ja.2007.92

15. Staerk, D.; Kesting, J. R.; Sairafianpour, M.; Witt, M.; Asili, J.; Emami, S. A.; Jaroszewski, J. W. Phytochemistry 2009, 70, 1055-1061. doi:10.1016/j.phytochem.2009.05.004

16. Wells, I. C. J. Biol. Chem. 1952, 196, 331-340.

17. Moon, S.-S.; Kang, P. M.; Park, K. S.; Kim, C. H. Phytochemistry 1996, 42, 365-368. doi:10.1016/0031-9422(95)00897-7

18. Gerth, K.; Trowitzsch, W.; Wray, V.; Höfle, G.; Irschik, H.; Reichenbach, H. J. Antibiot. 1982, 35, 1101-1103. doi:10.7164/antibiotics.35.1101

19. Itoh, J.; Amano, S.; Ogawa, Y.; Kodama, Y.; Ezaki, N.; Yamada, Y. J. Antibiot. 1980, 33, 377-382. doi:10.7164/antibiotics.33.377

20. Bisacchi, G. S. J. Med. Chem. 2015, 58, 4874-4882. doi:10.1021/jm501881c 
21. Michael, J. P. Nat. Prod. Rep. 2001, 18, 543-559. doi:10.1039/b005387m

22. Michael, J. P. Nat. Prod. Rep. 2003, 20, 476-493. doi:10.1039/b208140g

23. Michael, J. P. Nat. Prod. Rep. 2005, 22, 627-646. doi:10.1039/b413750g

24. Michael, J. P. Nat. Prod. Rep. 2007, 24, 223-246. doi:10.1039/b509528j

25. Michael, J. P. Nat. Prod. Rep. 2008, 25, 166-187. doi:10.1039/B612168N

26. Dekker, K. A.; Inagaki, T.; Gootz, T. D.; Huang, L. H.; Kojima, Y.; Kohlbrenner, W. E.; Matsunaga, Y.; McGuirk, P. R.; Nomura, E.; Sakakibara, T.; Sakemi, S.; Suzuki, Y.; Yamauchi, Y.; Kojima, N. J. Antibiot. 1998, 51, 145-152. doi:10.7164/antibiotics.51.145

27. Cheng, C.; Othman, E. M.; Reimer, A.; Grüne, M.; Kozjak-Pavlovic, V.; Stopper, H.; Hentschel, U.; Abdelmohsen, U. R. Tetrahedron Lett. 2016, 57, 2786-2789. doi:10.1016/j.tetlet.2016.05.042

28. Kunze, B.; Höfle, G.; Reichenbach, H. J. Antibiot. 1987, 40, 258-265. doi:10.7164/antibiotics.40.258

29. Vial, L.; Lepine, F.; Milot, S.; Groleau, M.-C.; Dekimpe, V.; Woods, D. E.; Deziel, E. J. Bacteriol. 2008, 190, 5339-5352. doi:10.1128/JB.00400-08

30. Long, R. A.; Qureshi, A.; Faulkner, D. J.; Azam, F. Appl. Environ. Microbiol. 2003, 69, 568-576. doi:10.1128/AEM.69.1.568-576.2003

31. Hertiani, T.; Edrada-Ebel, R. A.; van Soest, R. W. M.; Sudarsono, P. Indones. J. Pharm. 2008, 19, 128-136.

32. Heeb, S.; Fletcher, M. P.; Chhabra, S. R.; Diggle, S. P.; Williams, P.; Cámara, M. FEMS Microbial. Rev. 2011, 35, 247-274. doi:10.1111/j.1574-6976.2010.00247.x

33. Kitamura, S.; Hashizume, K.; lida, T.; Miyashita, E.; Shirahata, K.; Kase, H. J. Antibiot. 1986, 60, 1160-1166. doi:10.7164/antibiotics.39.1160

34. Moon, S. S.; Myung, E. J.; Cho, S. C.; Park, J. B.; Chung, B. J. Korean J. Pest. Sci. 2002, 6, 64-71.

35. Lépine, F.; Milot, S.; Déziel, E.; He, J.; Rahme, L. G. J. Am. Soc. Mass Spectrom. 2004, 15, 862-869. doi:10.1016/j.jasms.2004.02.012

36. Okada, B. K.; Wu, Y.; Mao, D.; Bushin, L. B.; Seyedsayamdost, M. R. ACS Chem. Biol. 2016, 11, 2124-2130. doi:10.1021/acschembio.6b00447

37. Wang, B.; Waters, A. L.; Sims, J. W.; Fullmer, A.; Ellison, S.; Hamann, M. T. Microb. Ecol. 2013, 65, 1068-1075. doi:10.1007/s00248-013-0213-4

38. Depke, T.; Franke, R.; Brönstrup, M. J. Chromatogr., B 2017, 1071, 19-28. doi:10.1016/j.jchromb.2017.06.002

39. Avendaño-Herrera, R.; Toranzo, A. E.; Magariños, B. Dis. Aquat. Org. 2006, 71, 255-266. doi:10.3354/dao071255

40. Oku, N.; Matsumoto, M.; Yonejima, K.; Tansei, K.; Igarashi, Y. Beilstein J. Org. Chem. 2014, 10, 1808-1816. doi:10.3762/bjoc.10.190

\section{License and Terms}

This is an Open Access article under the terms of the Creative Commons Attribution License

(http://creativecommons.org/licenses/by/4.0), which permits unrestricted use, distribution, and reproduction in any medium, provided the original work is properly cited.

The license is subject to the Beilstein Journal of Organic Chemistry terms and conditions:

(https://www.beilstein-journals.org/bjoc)

The definitive version of this article is the electronic one which can be found at:

doi:10.3762/bjoc. 14.122 